\title{
Evaluation of cranial base repair techniques utilizing a novel cadaveric CPAP model.
}

\author{
Chandala Chitguppi \\ Thomas Jefferson University \\ Ryan A. Rimmer \\ Thomas Jefferson University \\ Hermes G. Garcia \\ Thomas Jefferson University \\ Ian J. Koszewski \\ Thomas Jefferson University
}

Follow this and additional works at: https://jdc.jefferson.edu/otofp

Judd $\mathrm{H}$. Fastenberg

JPlats ofetfferscold diniverosity Commons

Let us know how access to this document benefits you

See next page for additional authors

\section{Recommended Citation}

Chitguppi, Chandala; Rimmer, Ryan A.; Garcia, Hermes G.; Koszewski, lan J.; Fastenberg, Judd H.; Nyquist, Gurston G.; Rosen, Marc R.; Huntley, Colin; Rabinowitz, Mindy R.; and Evans, James

J., "Evaluation of cranial base repair techniques utilizing a novel cadaveric CPAP model." (2019). Department of Otolaryngology - Head and Neck Surgery Faculty Papers. Paper 43.

https://jdc.jefferson.edu/otofp/43

This Article is brought to you for free and open access by the Jefferson Digital Commons. The Jefferson Digital Commons is a service of Thomas Jefferson University's Center for Teaching and Learning (CTL). The Commons is a showcase for Jefferson books and journals, peer-reviewed scholarly publications, unique historical collections from the University archives, and teaching tools. The Jefferson Digital Commons allows researchers and interested readers anywhere in the world to learn about and keep up to date with Jefferson scholarship. This article has been accepted for inclusion in Department of Otolaryngology - Head and Neck Surgery Faculty Papers by an authorized administrator of the Jefferson Digital Commons. For more information, please contact: JeffersonDigitalCommons@jefferson.edu. 
Authors

Chandala Chitguppi, Ryan A. Rimmer, Hermes G. Garcia, Ian J. Koszewski, Judd H. Fastenberg, Gurston G. Nyquist, Marc R. Rosen, Colin Huntley, Mindy R. Rabinowitz, and James J. Evans 
Title: Evaluation of Cranial Base Repair Techniques Utilizing a Novel Cadaveric CPAP Model Short running title: Post-operative CPAP and cranial base repair Authors: Chitguppi Chandala ${ }^{1}$, Rimmer Ryan $\mathrm{A}^{1}$, Garcia Hermes $\mathrm{G}^{2}$, Koszewski lan J $\mathrm{J}^{1}$, Fastenberg, Judd $\mathrm{H}^{1}$, Nyquist Gurston $\mathrm{G}^{1,2}$, Rosen Marc $\mathrm{R}^{1,2}$, Huntley Colin ${ }^{1}$, Rabinowitz Mindy $\mathrm{R}^{1,2^{*}}$, Evans James $\mathrm{J}^{1,2^{*}}$

1. Department of Otolaryngology - Head \& Neck Surgery, Thomas Jefferson University Hospital

2. Department of Neurological Surgery, Thomas Jefferson University Hospital

*Co-primary investigators

Institution where the work was conducted: Thomas Jefferson University Hospital, Philadelphia, PA.

Financial disclosure information: None

Conflict of Interest: None

\section{Corresponding author:}

Chandala Chitguppi, MD

Department of Otolaryngology - Head \& Neck Surgery,

Thomas Jefferson University Hospital

Philadelphia, PA

19107

Email address: chandala.chitguppi@jefferson.edu

Cell Number: 267-245-1959

Keywords: Obstructive Sleep apnea, CPAP, Airway, Skull base surgery, Cadaver 


\begin{abstract}
Introduction:

Although recent guidelines for obstructive sleep apnea recommend early post-operative use of continuous positive airway pressure (CPAP) following endonasal skull base surgery, time of initiation of CPAP is unclear. This study uses a novel, previously validated cadaveric model to analyze the pressures delivered to the cranial base and to evaluate the effectiveness of various repair techniques to withstand positive pressure.
\end{abstract}

Materials:

Skull base defects were surgically created in three fresh human cadaver heads and repaired using three commonly used repair techniques: (1) Surgicel ${ }^{\mathrm{TM}}$ onlay (2) Dural substitute underlay with dural sealant onlay and (3) Dural substitute underlay with Nasoseptal flap onlay with dural sealant. Pressure micro-sensors were placed in sphenoid sinus and sella, both proximal and distal to the repair, respectively. The effectiveness of each repair technique against various CPAP pressure settings $\left(5-20 \mathrm{~cm} \mathrm{H}_{2} \mathrm{O}\right)$ was analyzed.

\title{
Results:
}

Approximately $79-95 \%$ of positive pressure administered reached the sphenoid sinus. Sellar pressure levels varied significantly across the three repair techniques and were lowest following third technique. 'Breach' points (CPAP settings at which sellar repair was violated) were lowest for the first group. All three specimens showed a breach following first repair technique. For the second repair technique, only a single breach was created in one specimen at $20 \mathrm{~cm} \mathrm{H}_{2} \mathrm{O}$. No breaches were created in the third group.

\section{Conclusion:}

Different skull base repair techniques have varying ability to withstand CPAP. Both second and third repair techniques performed in a nearly similar fashion regarding their ability to withstand positive pressure ventilation. 


\section{Introduction}

Obstructive sleep apnea (OSA) is a common comorbid condition in various skull base tumors including functioning pituitary adenomas (1-4). Among the functioning pituitary adenomas, 34\% and $46 \%$ of patients with prolactinoma and corticotropinoma were found to suffer from OSA respectively $(3,4)$. Hormonal irregularities associated with functioning pituitary tumors contribute to obesity in some patients, putting them at risk for OSA $(1,4,5)$.

Comorbid OSA in patients undergoing endonasal skull base surgeries increases the risk of obstructive events and hypoxemia, as well as pulmonary and airway complications in the postoperative setting $(6,7)$. As a result, management of these patients represents a significant clinical challenge. Additionally, there is a general paucity of evidence-based recommendations for post-operative management of these patients. Although the American Society of Anesthesiology guidelines recommend early post-operative initiation of continuous positive airway pressure (CPAP) ventilation in OSA patients (8), further specifics especially with respect to its time of initiation and safety of usage are lacking. Furthermore, these guidelines do not distinguish between surgical patients for whom post-operative CPAP does and does not pose additional risk. For some patients undergoing pituitary and other skull base surgeries, positive pressure ventilation may in fact threaten the integrity of and cause breaches in skull base reconstructions leading to significant morbidity.

There is clear need for a more evidenced-based approach to post-operative management of patients with OSA undergoing skull base surgery, specifically concerning the use of CPAP. This study aims to help fill this void by objectively analyzing the positive pressures delivered to the skull base and by evaluating the effectiveness of three different sellar reconstructive techniques in a novel, previously validated cadaver CPAP model.

\section{Methods}




\section{A Preliminary set-up:}

Institutional review board approval was obtained. Fresh-frozen (unfixed) human cadaver heads were procured following institutional fresh tissue dissection laboratory protocol within 21-23 days of death and were stored at $5.6^{\circ} \mathrm{C}$ until use. The heads (specimens) were prepared as previously described (9). The laboratory was set up to simulate an operating room with a complete range of sinus and neurosurgical instruments including nitrogen tank to spray Duraseal $^{\mathrm{TM}}$ (Integra LifeScience Corp, Plainsboro, NJ), and standard endoscopy system using $0^{0}$ and $30^{\circ}, 4 \mathrm{~mm}$ rigid Storz endoscopes (Karl Storz, Tuttlingen, Germany) with digital recording capabilities.

\section{B Setting the CPAP machine:}

The CPAP machine was calibrated and titration was done as described previously (9). It was connected to the vertically-oriented head filled with water in the intracranial cavity and then run for one minute to confirm the absence of any pre-existing skull base defects. If any leaks are observed, they are sealed using bone wax or dural sealant as described previously (9). Pressure readings were displayed in millimeters of mercury $(\mathrm{mm} \mathrm{Hg})$ by the monitor and were converted to centimeters of water $\left(\mathrm{cm} \mathrm{H}_{2} \mathrm{O}\right)$ using a conversion factor of 1.36.

\section{C Creation of Skull base defect:}

Following confirmation of absence of pre-existing skull base defects, the CPAP machine was disconnected; water from the intra cranial cavity removed and the cadaveric specimens were placed at a $45^{\circ}$ angle and stabilized in such a way to anatomically mimic typical surgical positioning for endoscopic sinus surgery. Endoscopic endonasal and sellar dissection followed the steps as previously described (9).The current study protocol is given below (Figure 1). Additional details regarding skull base defect creation are as follows.

Under endoscopic visualization, the sellar dura was opened by a cruciate incision with a retractable knife. A complete hypophysectomy was performed. Diaphragm sellae was now clearly visualized endonasally. The surgeon confirmed this (location of diaphragm sellae) from 
the intra cranial end by using the glow of the endoscope (placed intra-nasally) as a guide. A specialized neurosurgical spatula, with a flat end measuring $3 \mathrm{~mm}$ in diameter (in order to maintain consistency of defect size) was utilized to create a single defect in the center of the sellar diaphragm in each specimen. The spatula was inserted through the nasal cavity under direct endoscopic guidance. After creation of the defect, the specimen was placed back in the vertical position and water was replaced into the intracranial cavity until the skull base was fully submerged. The CPAP machine was then reconnected and run for one minute to confirm presence of the diaphragm sella defect we created (seen as air bubbles in the intracranial cavity arising from the diaphragm sella).

\section{D Placement of pressure micro-sensors:}

Codman Micro-sensor ${ }^{\mathrm{TM}}$ ventricular catheter kits (Codman \& Shurtieff, Raynham, MA) and monitors were used to measure the sphenoid and sellar pressures. The outer sheaths were removed to decrease the sensor diameter and to facilitate easier intranasal manipulation within the specimens. Following creation of the defect in the diaphragm sella, micro-sensors were placed in both the sella (from the intracranial cavity) and sphenoid cavity (endonasally) such that they were proximal and distal to the repair, respectively (Image 1). The sensors were zeroed prior to insertion (the intracranial submerged sensor was zeroed in water and the endonasal sensor was zeroed in air). Placement in the sphenoid sinus cavity was performed as previously described (9). The sellar sensor was positioned through the intracranial cavity (Images 2A, 2B, 2C). Proper placement was endoscopically confirmed and then the sensor was fixed to the nearby dura with Vicryl 4-0 suture in order to stabilize its position throughout the experiment. Care was taken to keep the sensors 'free' within the sphenoid cavity and sella, avoiding the tip of the sensors from pressing on any adjacent structures.

\section{E Repair techniques and CPAP administration:}

Three commonly used but distinct cranial base repair techniques were analyzed in succession on each of the three specimens. Each repair technique is similar to the skull base reconstructive algorithm used for patients at our institution (10). 
Initially, two layers of Surgicel ${ }^{\mathrm{TM}}$ (oxidized cellulose) (Ethicon, Inc., Somerville, NJ) were placed in an onlay fashion over the created sellar dural defect (Image 3). The CPAP machine was connected and then turned on starting at a setting of $5 \mathrm{~cm} \mathrm{H}{ }_{2} \mathrm{O}$. The machine was run for 2 minutes. If no 'breach' (defined below) was detected, the pressure was increased by increments of $3 \mathrm{~cm} \mathrm{H} \mathrm{H}_{2} \mathrm{O}$ every 2 minutes until a maximum of $20 \mathrm{~cm} \mathrm{H} \mathrm{H}_{2} \mathrm{O}$ pressure had been reached. CPAP administration was discontinued when the pressure had reached $20 \mathrm{~cm} \mathrm{H}_{2} \mathrm{O}$. If a 'breach' was detected during pressure administration, then the CPAP levels at which 'breach' occurred were recorded. The remaining details are provided in the study protocol (Figure 1). Pressure readings from the micro-sensors were recorded for each CPAP setting between 5-20 $\mathrm{cm} \mathrm{H}_{2} \mathrm{O}$. This pressure range was chosen because it corresponds to the recommended CPAP range as per guidelines from American Academy of Sleep Medicine in adult OSA patients (11).

Subsequently, Surgicel ${ }^{\mathrm{TM}}$ was gently and fully removed and then, a second repair technique (Image 4) was performed by placing a dural substitute, Durepair ${ }^{\mathrm{TM}}$ inlay (Medtronic Inc., Minneapolis, MN) followed by dural sealant, Duraseal ${ }^{\mathrm{TM}}$ (Integra LifeScience Corp., Plainsboro, NJ) onlay placement using a nitrogen tank. Pressure recordings were again obtained in a similar fashion.

The third and final repair technique was then performed, with the pre-existing dural substitute inlay in-situ (who's proper placement was confirmed endoscopically), followed by placement of a nasoseptal flap (NSF) with subsequent dural sealant placement using a nitrogen tank (Image 5). The NSF was harvested from the side of the nose with more space.

Maximum CPAP administration time for each repair technique was 12 minutes, not including the initial time for setup and identification of previously existing leaks. During the experiment, it should be noted that both sphenoid and sellar pressures were simultaneously recorded at each CPAP pressure setting ( 5 to $20 \mathrm{~cm} \mathrm{H}_{2} \mathrm{O}$ ) for each specimen. 


\section{F Defining 'breach' and 'breach points':}

For the purpose of our study, we defined the term 'breach' as a loss in the integrity of the sellar repair as demonstrated by sudden appearance of air bubbles arising from the sellar area (identified in the intracranial water level, visualized from the intracranial cavity) when the CPAP system was connected (Image 6). A 'breach point' was defined as the CPAP setting at which 'breach' occurred and it represented the end-point for CPAP administration.

\section{G Statistical analysis:}

Descriptive statistics for continuous variables included calculation of mean, percentage and proportion. Analysis for continuous data with three variables was performed using ANOVA test to explore for significant difference between different groups. All statistical tests were evaluated at alpha level of 0.05 .

\section{Results}

The three specimens will be referred to as ' 1 ', ' 2 ' and ' 3 ' respectively, in the following sections. Repair using Surgicel ${ }^{\mathrm{TM}}$ will be described as first repair technique, dural substitute with dural sealant as second repair technique and finally NSF with dural substitute/ dural sealant as third repair technique.

\section{A. Comparison of Sphenoid pressure readings:}

The details of the sphenoid pressure readings are shown in Table 1. Approximately 79-95\% of CPAP administered $\left(\mathrm{cm} \mathrm{H}_{2} \mathrm{O}\right)$ at the level of external nares reached the level of the sphenoid sinus cavity. There was no statistical difference $(p>0.05)$ in positive pressure delivered to the sphenoid sinus based on repair technique. In most instances, increase in CPAP setting resulted in proportionately higher pressure transmitted to the sphenoid sinus

\section{B. Comparison of sellar pressure readings:}

The details of the sellar pressure readings are shown in Table 2. Sellar pressure levels were compared between the repair techniques and were found to be distinct among the three repair 
techniques. Significant difference was noted in two specimens $(p<0.0001,0.0004)$ while the difference approached level of significance in the third $(p=0.089)$. For two of the three specimens, no amount of pressure was transmitted to the sella following the second and third repair techniques. The sellar pressures readings for the range of CPAP settings tested in specimen 1, 2 and 3 are depicted in Fig. 2A, Fig. 2B, and Fig. 2C respectively.

\section{C. Proportion of sellar pressure:}

Although between the three repair techniques, dissimilar sellar pressure recording were found, it was not known whether this finding was consistent across all CPAP settings. Therefore, the proportion of CPAP reaching the sella was analyzed for each CPAP setting and the results have been depicted in Figure 3. Proportion of sellar pressure was calculated as the sellar pressure reading $\left(\mathrm{cm} \mathrm{H}_{2} \mathrm{O}\right)$ divided by the total CPAP setting at external nares $\left(\mathrm{cm} \mathrm{H}_{2} \mathrm{O}\right)$ multiplied by 100, expressed as a percentage. For instance, the proportion of sellar pressure for a sellar pressure reading of $1 \mathrm{~cm} \mathrm{H} \mathrm{C}_{2} \mathrm{O}$ at CPAP setting of $5 \mathrm{~cm} \mathrm{H} \mathrm{H}_{2} \mathrm{O}$ at external nares was $20 \%$. It can be noted that across all CPAP levels, the second and third repair techniques showed almost similar performance and transmitted lower proportion of pressure to sella as compared to the first repair technique.

\section{D Analysis of breach}

'Breach points' including corresponding sphenoid and sellar pressure recordings were analyzed for the three repair techniques (Table 3). Surgicel ${ }^{\mathrm{TM}}$ had the lowest 'breach point' with all three specimens showing a 'breach' (seen as yellow boxes in figure 2A, 2B and 2C), while no breaches were detected with the third repair technique

\section{Discussion}

There is a clear need for an evidence-based approach to the management of patients with OSA undergoing skull base surgery, specifically regarding CPAP use. This is the first study of its kind

to objectively analyze the dynamics of positive pressure ventilation at the level of the skull base 
and, furthermore, to evaluate the ability of different skull base reconstructions to withstand positive pressure ventilation in a validated cadaveric model (9).

The three repair techniques used in this study were selected because they are all part of the reconstructive algorithm used at this institution. Although many repair techniques have been described in the literature for skull base defects (12), the three repair techniques chosen in our study represent a spectrum of repair techniques that are appropriate for a wide range of different clinical situations. These repair techniques can be utilized in both small and large skull base defects, defects in the absence of CSF leak, as well as those with either a low-flow or highflow CSF leak. Furthermore, each repair is distinct from the other with respect to materials and complexity of placement, with the Surgicel ${ }^{\mathrm{TM}}$ onlay representing both the easiest and most inexpensive method and NSF representing the more complicated technique. Given the increased focus on both cost and total operative time, the modern skull base surgeon must consider these concerns.

As demonstrated by the sellar pressure recordings (which were located distal or 'behind' the repair), we found that different repair techniques offered varying degrees of 'protection'. This is in contrast to sphenoid pressure recordings (which were proximal to the repair), which were approximately equivalent among the repair techniques. This shows consistency of the pressure delivered to the sphenoid sinus proportionate to the CPAP setting unlike sellar pressure that varied with repair techniques. As could be expected, the first Surgicel ${ }^{\mathrm{TM}}$ onlay repair was able to withstand the least amount of positive pressure. This may be related to the porous nature of Surgice ${ }^{\mathrm{TM}}$, as well as its lack of bulk. Furthermore, without addition of a 'binding' agent such as dural sealant, lack of adherence to the edges of the defect may have been contributory. As expected, the third repair technique involving dural substitute underlay, NSF and dural sealant was able to withstand the greatest amount of positive pressure with no breaches detected in any of the specimens at any pressure administered up to $20 \mathrm{~cm} \mathrm{H}_{2} \mathrm{O}$. Perhaps most notably were the results from the second repair technique involving dural substitute underlay and dural sealant, which performed in nearly an equivalent manner to the third repair, with only a single 
breach in one of the specimens at a CPAP setting of $20 \mathrm{~cm} \mathrm{H} \mathrm{H}_{2} \mathrm{O}$. The intra-sellar pressures were also identical in 2 out of 3 specimens for all CPAP settings in both the second and third repair techniques.

Although the findings suggest that, the first repair technique was not able to withstand positive pressure as effectively as the second and third repair techniques, it is worthwhile to analyze the CPAP settings at which breach occurred following the first repair technique. Studies analyzing the pressure profiles of OSA patients using auto-CPAP devices usually have median CPAP settings of 5.2-7.2 $\mathrm{cm} \mathrm{H}_{2} \mathrm{O}$ (13). This range of CPAP setting is considerably lower than that noted in our study, at which breach is seen following the first repair technique $\left(11-14 \mathrm{~cm} \mathrm{H}_{2} \mathrm{O}\right)$. Therefore, although Surgice ${ }^{\mathrm{TM}}$ had poorer endurance against positive pressure ventilation, it may in fact be just as effective as the other two techniques in OSA patients who require lower pressure profile on CPAP in comparison to second and third technique.

Unlike Surgicel ${ }^{\mathrm{TM}}$, second and third repair techniques were found to offer more robust protection against most CPAP settings even in the immediate post-operative period. At least initially, this degree of integrity may be related to use of an underlay repair technique or due to the use of dural sealants which provide a seal along the edges of the repair, before there has been adequate time for tissue integration and vascularization. The nearly equivocal performance of the second repair technique, in comparison to the third, supports the utilization of reconstructive algorithms that appropriately utilize dural substitutes such as Durepair ${ }^{\mathrm{TM}}$, and furthermore that reserve the use of a NSF for only those select clinical situations, such as large defects with associated high-flow CSF leak, that cannot be addressed with other repair techniques.

The management of OSA and the decision to start CPAP post-operatively must be tailored to each patient based on the severity of his/her disease, as well as the details of surgery including the kind of skull base reconstruction. Based on the findings of this study, it is likely safest to initiate CPAP in those patients who have undergone a 'sturdier' skull base reconstruction 
capable of withstanding positive pressure, such as those with underlay grafts, NSF, and/or dural sealants. However, further research in the live subjects is advised to confirm the same. Furthermore, as evidenced by elevated pressure recordings for higher CPAP settings, initiation of CPAP at relatively low pressures is likely the safest option.

We acknowledge the limitations of the study, especially those inherent to a cadaveric model. This includes the absence of elements such as postoperative edema, crusting, lung compliance and brain weight, all of which could impact the dynamics of positive pressure at the level of the skull base. Additionally, patient related factors like BMI, coughing, activity level and position of head cannot be accounted for. The cadaveric model is essentially a 'closed-loop system' and this may have impacted pressure levels measured. Furthermore, in this study, positive pressure was only administered for 25-35 minutes per specimen. This is in contrast to the several hours of CPAP usage by OSA patients each night. However, studying the effect of CPAP administration for prolonged duration (4-6 hours at least 5 times a week) is not feasible in a laboratory set up. Furthermore, it is possible that following vascularization and adherence of the repaired layer, the endurance of the repair technique against positive pressure ventilation continues to increase, in which case the duration of CPAP usage may not affect the repair.

In spite of the above limitations, this study represents the first of its kind. Future prospective, large-scale studies in live patients are underway at this institution and are necessary to investigate the safety of post-operative CPAP use and to develop evidence-based guidelines for patients undergoing skull base surgery with comorbid OSA.

\section{Conclusion}

Distinct skull base repair techniques have varying ability to withstand positive pressure ventilation. Repairs involving dural substitute-dural sealant, with or without NSF, demonstrated similar ability to withstand CPAP. Decisions regarding use of post-operative CPAP should be individualized based on the clinical situation including both the severity of comorbid OSA and surgical details including the type of skull base reconstruction used. 


\section{References}

1. Friedel ME, Johnston DR, Singhal S, et al. Airway management and perioperative concerns in acromegaly patients undergoing endoscopic transsphenoidal surgery for pituitary tumors. Otolaryngol Head Neck Surg 2013;149:840-4

2. Murali R, Douyon P, Omeis I. Large anterior skull base lesions in patients presenting with obstructive sleep apnea. Report of two cases and review of the literature. J Neurosurg. 2004;101(1):163-6.

3. Sze L, Schmid C, Bloch KE, et al. Effect of transsphenoidal surgery on sleep apnoea in acromegaly. Eur J Endocrinol 2007;156:321-9.

4. Barbosa FR, Silva CM, Lima GA, et al. Prevalence of obstructive sleep apnea in patients with prolactinoma before and after treatment with dopamine agonists. Pituitary 2014;17:441-9.

5. Smith M, Hirsch NP. Pituitary disease and anaesthesia. Br J Anaesth 2000;85:3-14.

6. Huyett P, Soose RJ, Schell AE, et al. Risk of Postoperative Complications in Patients with Obstructive Sleep Apnea following Skull Base Surgery. Otolaryngol Head Neck Surg. 2018;158(6):1140-1147.

7. Chung SY, Sylvester MJ, Patel VR, et al. Impact of obstructive sleep apnea in transsphenoidal pituitary surgery: An analysis of inpatient data. Laryngoscope. 2018;128(5):1027-1032. 
8. Gross JB, Bachenberg KL, Benumof JL, et al. Practice guidelines for the perioperative management of patients with obstructive sleep apnea: a report by the American Society of Anesthesiologists Task Force on Perioperative Management of patients with obstructive sleep apnea. Anesthesiology 2006;104:1081-93 [quiz 117-8].

9. Rimmer RA, Chitguppi C, Garcia HG, et al. A cadaveric model for measuring sinonasal continuous positive airway pressure-a proof-of-concept study. Int Forum Allergy Rhinol. 2018 [Epub ahead of print]

10. Gill KS, Hsu D, Tassone P, et al. Postoperative cerebrospinal fluid leak after microvascular reconstruction of craniofacial defects with orbital exenteration. Laryngoscope.2017;127(4):835-841.

11. Kushida CA; Chediak A; Berry RB; Brown LK; Gozal D; Iber C; Parthasarathy S; Quan SF; Rowley JA; Positive Airway Pressure Titration Task Force of the American Academy of Sleep Medicine. Clinical guidelines for the manual titration of positive airway pressure in patients with obstructive sleep apnea. J Clin Sleep Med 2008;4(2):157-171.

12. Soudry E, Turner JH, Nayak JV, Hwang PH. Endoscopic reconstruction of surgically created skull base defects: a systematic review. Otolaryngol Head Neck Surg. 2014;150(5):730-8.

13. Hertegonne KB, Proot PM, Pauwels RA, Pevernagie DA. Comfort and pressure profiles of two auto-adjustable positive airway pressure devices: a technical report. Respir Med. 2003;97(8):903-8. 


\section{Appendix:}

Fig 1 depicts the flowchart showing the study protocol.

Three repair techniques are performed in succession and pressure readings at sphenoid and sella are recorded simultaneously.

Fig 2A depicts the change in sellar pressure readings with change in CPAP settings in specimen 1.

$\mathrm{X}$-axis denotes changes in CPAP settings and Y-axis denotes changes in sellar pressure readings. The changes that occurred on performing the three repair techniques have been denoted using colored horizontal lines. Green horizontal line denotes sellar pressure readings following Surgicel ${ }^{\mathrm{TM}}$ repair, blue is following dural substitute (Durepair ${ }^{\mathrm{TM}}$ ) repair and red is following NSF repair. The values in the yellow boxes denote the sellar pressure readings at which 'breach' occurred for that particular repair technique. Absence of yellow boxes for a particular horizontal line denotes absence of breach in any of the CPAP settings tested for that particular repair technique (For instance in specimen 1, no breach was noted after NSF repair).

Fig 2B depicts the change in sellar pressure readings with change in CPAP settings in specimen $\underline{2}$.

$\mathrm{X}$-axis denotes changes in CPAP settings and $\mathrm{Y}$-axis denotes changes in sellar pressure readings. The changes that occurred with the three repair techniques have been denoted using colored horizontal lines. Green horizontal line denotes sellar pressure readings following Surgicel ${ }^{\mathrm{TM}}$ repair, blue is following dural substitute (Durepair ${ }^{\mathrm{TM}}$ ) repair and red is following NSF repair. The values in the yellow boxes denote the sellar pressure readings at which 'breach' occurred in the repaired defect for that particular repair technique. Absence of yellow boxes for a particular horizontal line denotes absence of breach in any of the CPAP settings tested for that particular repair technique (For instance in specimen 2, no breach was noted after Durepair ${ }^{\mathrm{TM}}$ and NSF repair).

Fig $2 \mathrm{C}$ depicts the change in sellar pressure readings with change in CPAP settings in specimen $\underline{3 .}$

$\mathrm{X}$-axis denotes changes in CPAP settings and $\mathrm{Y}$-axis denotes changes in sellar pressure readings. The changes that occurred with the three repair mechanisms have been denoted using colored horizontal lines. Green horizontal line denotes sellar pressure changes following Surgicel ${ }^{\mathrm{TM}}$ repair, blue is following dural substitute (Durepair ${ }^{\mathrm{TM}}$ ) repair and red is following NSF repair. The values in the yellow boxes denote the sellar pressure readings at which 'breach' occurred in the repaired defect for that particular repair technique. Absence of yellow boxes for a particular horizontal line denotes absence of breach in any of the CPAP settings tested for that particular 
repair technique (For instance in specimen 3, no breach was noted after Durepair ${ }^{\mathrm{TM}}$ and NSF repair).

Fig 3 depicts the proportion of sellar pressure recorded following three repair techniques.

$\mathrm{X}$-axis determines the mean proportion of sellar pressure (in percentage) at each level of CPAP setting (considering respective CPAP setting delivered at the external nares as $100 \%$ ). Y-axis denotes different CPAP settings tested $(5-20 \mathrm{~cm} \mathrm{H} 2 \mathrm{O})$. The green bar represents mean proportion of sella pressure following Surgicel ${ }^{\mathrm{TM}}$, the blue bar is following dural substitute (Durepair $^{\mathrm{TM}}$ ) and red bar, following NSF repair techniques. 


\section{Images:}

\section{Image 1: Placement of sphenoid sensor}

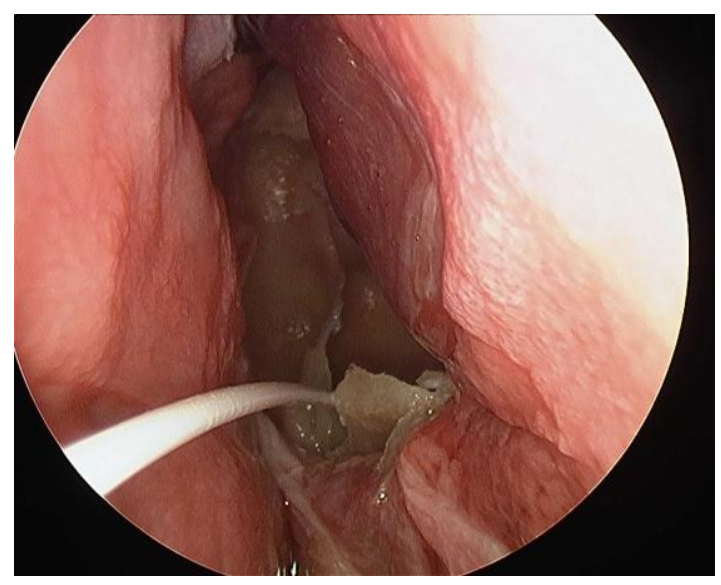

Image 2A: Placement of sellar sensor

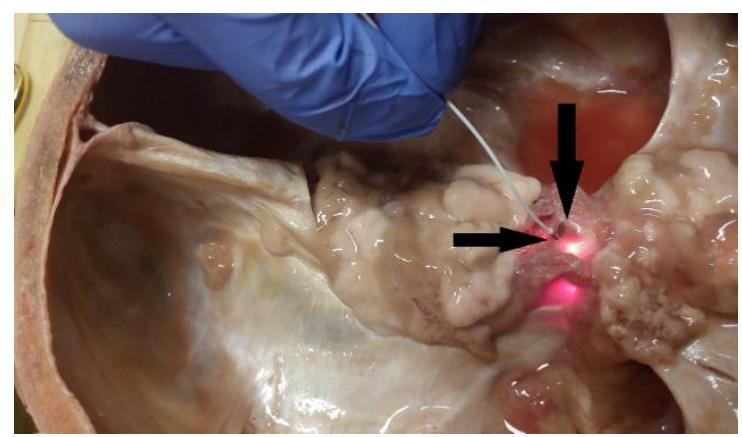

The arrows denotes to the tip of pressure sensor inserted into sellar defect

Image 2B: Fixation of sellar sensor

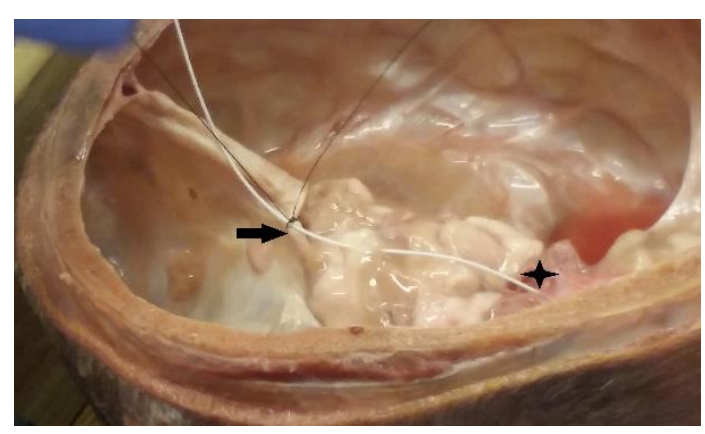

The arrow denotes fixation of pressure sensor to the nearby dura. The star denotes tip of sellar senor inserted into the sella through the defect. 
Image 2C: Endoscopic view of the final position of sellar sensor

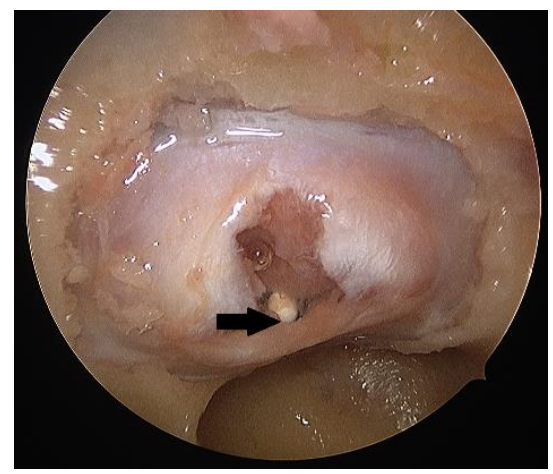

The arrow denotes to the tip of pressure sensor inserted into sellar defect

Image 3: Endoscopic view of sellar defect after first repair technique using Surgicel ${ }^{\mathrm{TM}}$

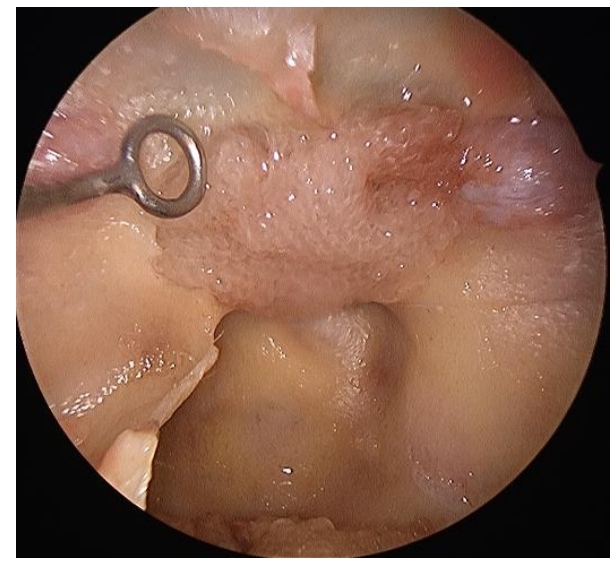

Image 4: Endoscopic view of sellar defect after second repair technique using dural substitute and dural sealant

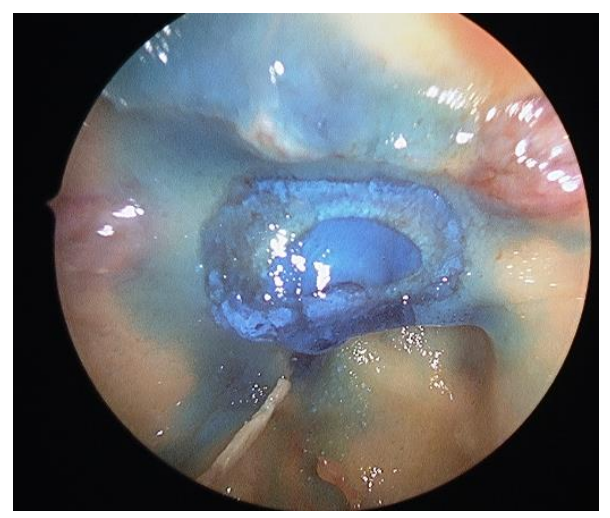


Image 5: Endoscopic view of sellar defect after second repair technique using nasoseptal flap and dural sealant

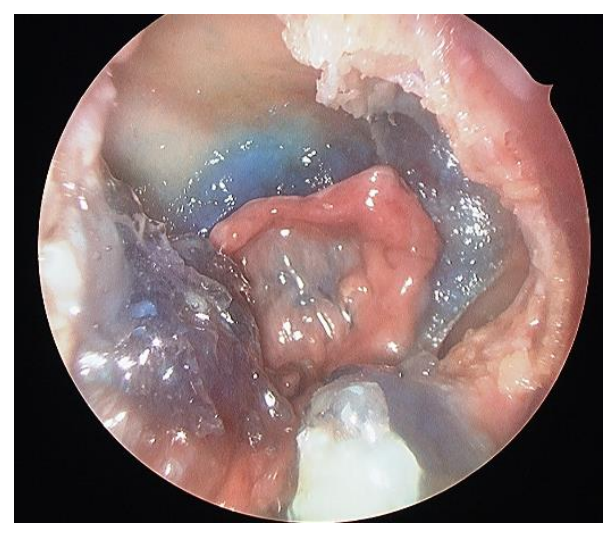

Image 6: Detection of 'breach' in skull base

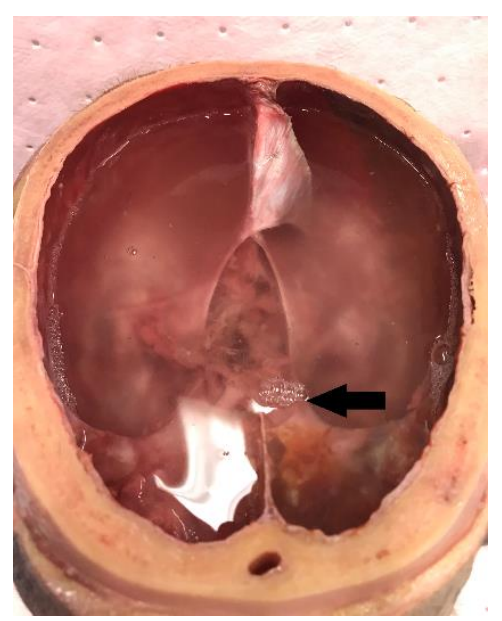

The arrow points to the air bubbles seen arising from sellar area during CPAP usage 
Tables:

Table 1: Comparison of Sphenoid pressure readings after three repair techniques

\begin{tabular}{|c|c|c|c|c|c|}
\hline & $\begin{array}{l}\text { CPAP level } \\
\left(\mathrm{cm} \mathrm{H} \mathrm{H}_{2} \mathrm{O}\right)\end{array}$ & $\begin{array}{l}\text { Surgicel }^{\mathrm{TM}} \\
\left(\mathrm{cm} \mathrm{H} \mathrm{H}_{2} \mathrm{O}\right)\end{array}$ & $\begin{array}{l}\text { Dural substitute } \\
\left(\mathrm{cm} \mathrm{H}_{2} \mathrm{O}\right)\end{array}$ & $\begin{array}{l}\text { NSF } \\
\left(\mathrm{cm} \mathrm{H} \mathrm{H}_{2} \mathrm{O}\right)\end{array}$ & P values \\
\hline \multirow{6}{*}{ Specimen 1} & 5 & 4.08 & 4.08 & 4.08 & \multirow{6}{*}{0.82} \\
\hline & 8 & 6.8 & 6.8 & 6.8 & \\
\hline & 11 & $9.52^{\#}$ & 9.52 & 9.52 & \\
\hline & 14 & 12.24 & 10.88 & 12.24 & \\
\hline & 17 & 13.6 & 12.24 & 14.96 & \\
\hline & 20 & 13.6 & $14.96^{\#}$ & 17.68 & \\
\hline \multirow{6}{*}{ Specimen 2} & 5 & 4.08 & 4.08 & 4.08 & \multirow{6}{*}{0.98} \\
\hline & 8 & 6.8 & 6.8 & 5.44 & \\
\hline & 11 & 9.52 & 10.88 & 10.88 & \\
\hline & 14 & $12.24^{\#}$ & 13.6 & 13.6 & \\
\hline & 17 & 14.96 & 16.32 & 16.32 & \\
\hline & 20 & 19.04 & 19.04 & 17.68 & \\
\hline \multirow{6}{*}{ Specimen 3} & 5 & 4.08 & 4.08 & 4.08 & \multirow{6}{*}{1.000} \\
\hline & 8 & 6.8 & 5.44 & 6.8 & \\
\hline & 11 & $10.88^{\#}$ & 10.88 & 10.88 & \\
\hline & 14 & 13.6 & 13.6 & 13.6 & \\
\hline & 17 & 16.32 & 16.32 & 16.32 & \\
\hline & 20 & 19.04 & 19.04 & 19.04 & \\
\hline
\end{tabular}

\# Denotes sphenoid pressures at which breach was noted 
Table 2: Comparison of sellar pressure readings after three repair techniques

\begin{tabular}{|c|c|c|c|c|c|}
\hline & CPAP level & Surgicel $^{\mathrm{TM}}$ & $\begin{array}{l}\text { Dural substitute } \\
\left(\mathrm{cm} \mathrm{H} \mathrm{H}_{2} \mathrm{O}\right)\end{array}$ & $\begin{array}{l}\text { NSF } \\
\text { (cm Hal }\end{array}$ & P values \\
\hline \multirow{6}{*}{ Specimen 1} & 5 & 1.36 & 1.36 & 1.36 & \multirow{6}{*}{0.089} \\
\hline & 8 & 1.36 & 1.36 & 1.36 & \\
\hline & 11 & $2.72^{\#}$ & 1.36 & 1.36 & \\
\hline & 14 & 2.72 & 2.72 & 1.36 & \\
\hline & 17 & 10.88 & 2.72 & 1.36 & \\
\hline & 20 & 10.88 & $4.08^{\#}$ & 1.36 & \\
\hline \multirow{6}{*}{ Specimen 2} & 5 & 2.72 & 0 & 0 & \multirow{6}{*}{$<.0001$} \\
\hline & 8 & 2.72 & 0 & 0 & \\
\hline & 11 & 2.72 & 0 & 0 & \\
\hline & 14 & $5.44^{\#}$ & 0 & 0 & \\
\hline & 17 & 5.44 & 0 & 0 & \\
\hline & 20 & 6.8 & 0 & 0 & \\
\hline \multirow{6}{*}{ Specimen 3} & 5 & 1.36 & 0 & 0 & \multirow{6}{*}{0.00045} \\
\hline & 8 & 1.36 & 0 & 0 & \\
\hline & 11 & $9.52^{\#}$ & 0 & 0 & \\
\hline & 14 & 12.24 & 0 & 0 & \\
\hline & 17 & 12.24 & 0 & 0 & \\
\hline & 20 & 13.6 & 0 & 0 & \\
\hline
\end{tabular}

\# Denotes sellar pressures at which breach was noted 
Table 3: Analysis of breach

\begin{tabular}{|l|l|l|l|}
\hline & Surgicel & Dural substitute & NSF \\
\hline No. of specimens which showed breach & $3 / 3$ & $1 / 3$ & $0 / 3$ \\
\hline Max CPAP tolerated $\left(\mathrm{cm} \mathrm{H}_{2} \mathrm{O}\right)$ & 12 & 20 & $20^{*}$ \\
\hline Mean sellar pressure reading $\left(\mathrm{cm} \mathrm{H}_{2} \mathrm{O}\right)$ & 5.89 & 2.72 & $0.45^{*}$ \\
\hline Proportion of sellar pressure & & & $2.26 \%^{*}$ \\
\hline
\end{tabular}

* Without breach in any of the specimens even at maximum pressure of $20 \mathrm{~cm} \mathrm{H}_{2} \mathrm{O}$. 
Fig 1 depicts the flowchart showing the study protocol.

Step 1: Confirm absence of pre-existing skull base defects

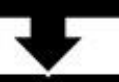

Step 2: Endoscopic sphenoidotomy with removal of sellar face and cruciate dural incision

Step 3: Hypophysectomy followed by stab incision in diaphragm sellae

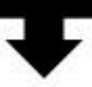

Step 4: Presence of diaphragmatic defect confirmed

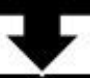

Step 5: Pressure sensor placement in sphenoid and sella

Step 6: Repair technique \#1

2

Step 7: CPAP started at $5 \mathrm{~cm} \mathrm{H}_{2} \mathrm{O}$ and increased by 3 units every 2 min

Step 8: Discontinue at $20 \mathrm{~cm} \mathrm{H}_{2} \mathrm{O}$

1

Step 9: Gently remove Surgicel ${ }^{\mathrm{TM}}$

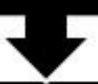

Step 10: Repair technique \#2. Repeat step 7 and 8

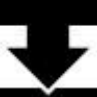

Step 11: Repair technique \#3. Repeat Step 7 and 8

Three repair techniques are performed in succession and pressure readings at sphenoid and sella are recorded simultaneously. 
Fig 2A depicts the change in sellar pressure readings with change in CPAP settings in specimen 1.

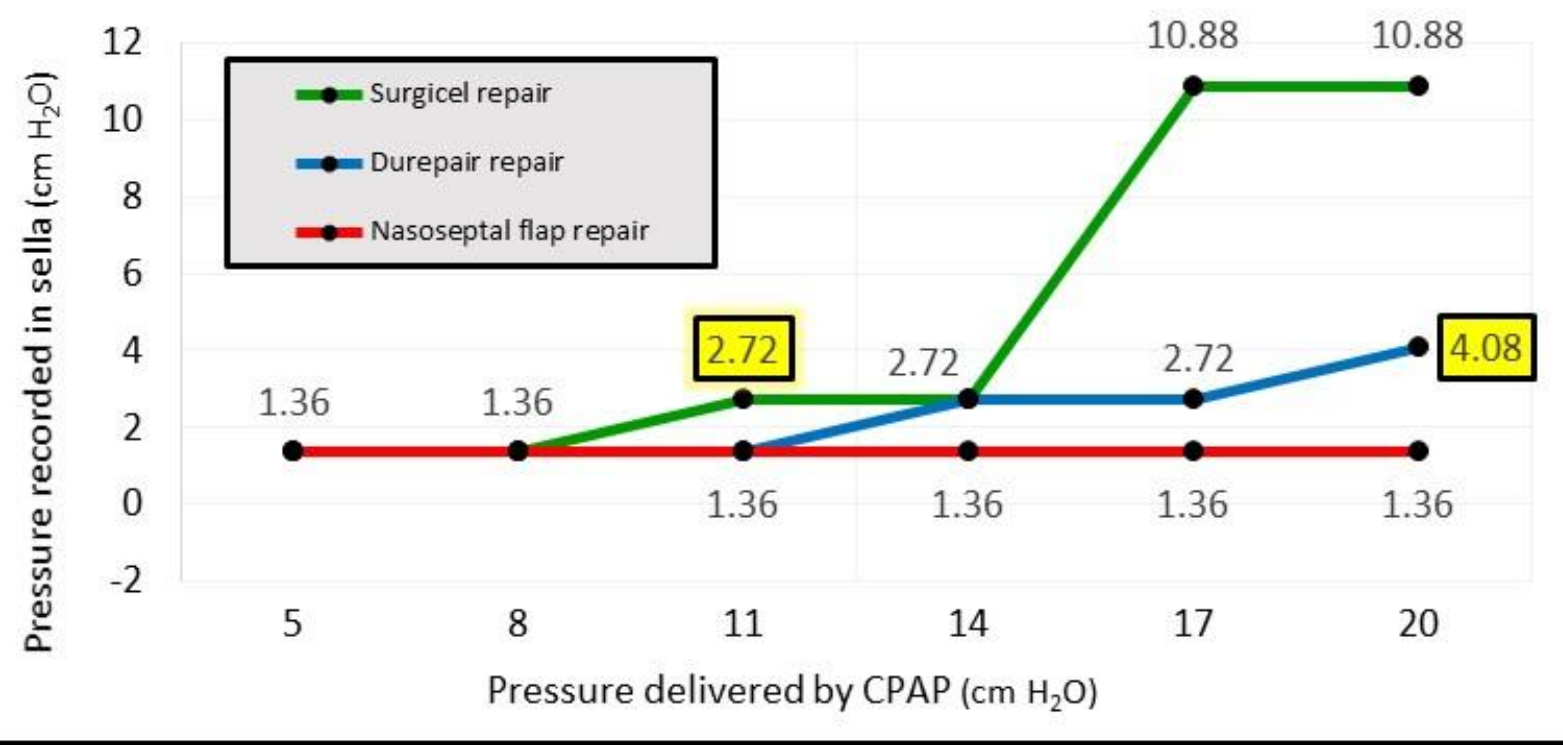

$\mathrm{X}$-axis denotes changes in CPAP settings and $\mathrm{Y}$-axis denotes changes in sellar pressure readings. The changes that occurred on performing the three repair techniques have been denoted using colored horizontal lines. Green horizontal line denotes sellar pressure readings following Surgicel ${ }^{\mathrm{TM}}$ repair, blue is following dural substitute (Durepair ${ }^{\mathrm{TM}}$ ) repair and red is following NSF repair. The values in the yellow boxes denote the sellar pressure readings at which 'breach' occurred for that particular repair technique. Absence of yellow boxes for a particular horizontal line denotes absence of breach in any of the CPAP settings tested for that particular repair technique (For instance in specimen 1, no breach was noted after NSF repair). 
Fig 2B depicts the change in sellar pressure readings with change in CPAP settings in specimen 2.

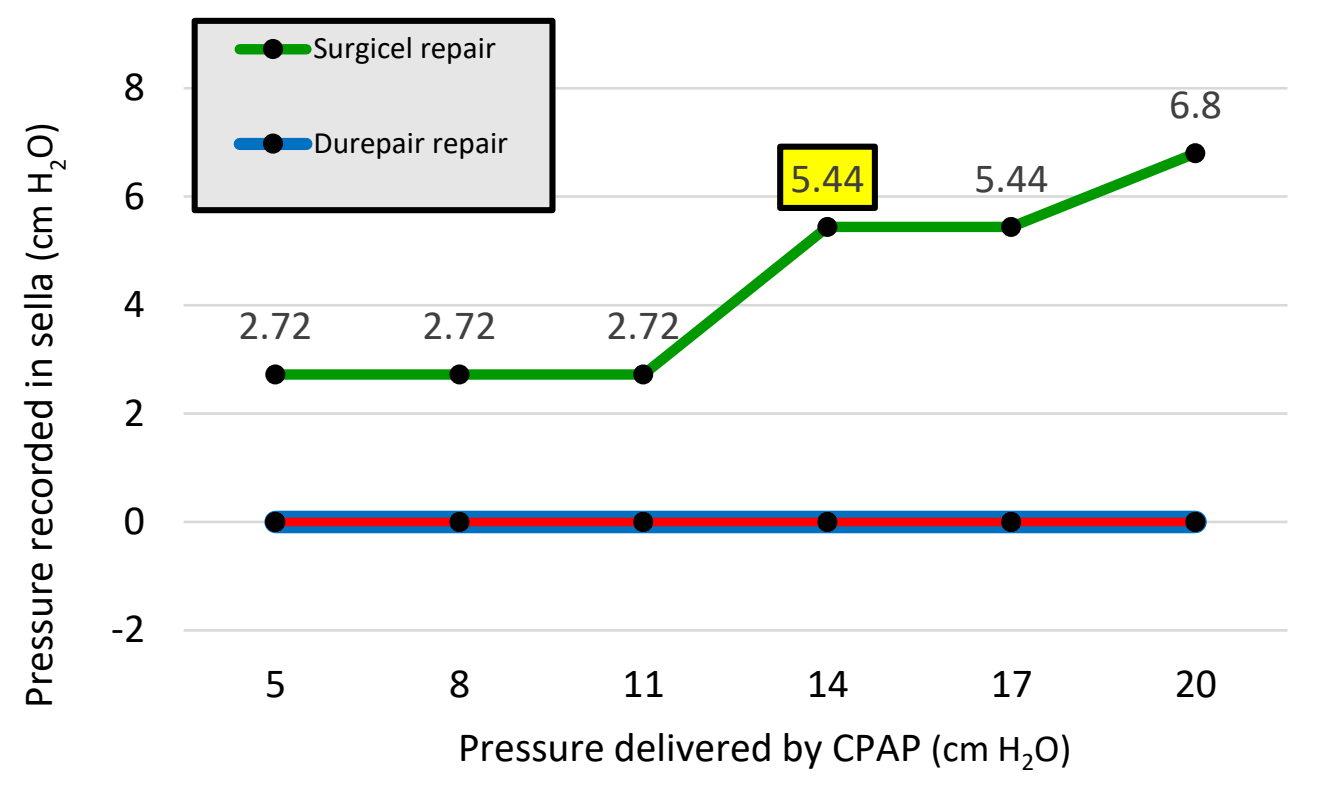

$\mathrm{X}$-axis denotes changes in CPAP settings and $\mathrm{Y}$-axis denotes changes in sellar pressure readings.

The changes that occurred with the three repair techniques have been denoted using colored horizontal lines. Green horizontal line denotes sellar pressure readings following Surgicel ${ }^{\mathrm{TM}}$ repair, blue is following dural substitute (Durepair ${ }^{\mathrm{TM}}$ ) repair and red is following NSF repair. The values in the yellow boxes denote the sellar pressure readings at which 'breach' occurred in the repaired defect for that particular repair technique. Absence of yellow boxes for a particular horizontal line denotes absence of breach in any of the CPAP settings tested for that particular repair technique (For instance in specimen 2, no breach was noted after Durepair ${ }^{\mathrm{TM}}$ and NSF repair). 
Fig $2 \mathrm{C}$ depicts the change in sellar pressure readings with change in CPAP settings in specimen 3.

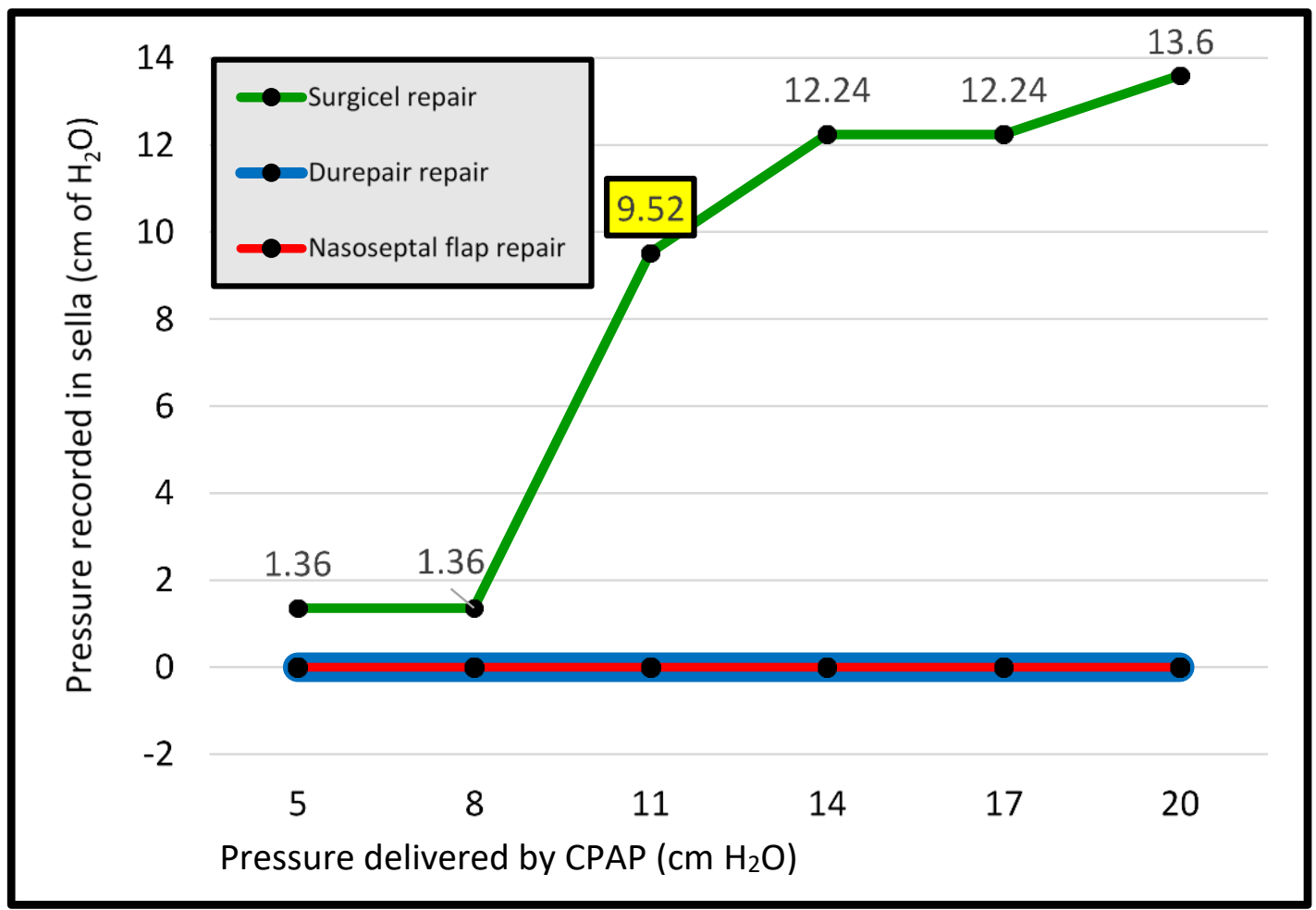

$\mathrm{X}$-axis denotes changes in CPAP settings and $\mathrm{Y}$-axis denotes changes in sellar pressure readings.

The changes that occurred with the three repair mechanisms have been denoted using colored horizontal lines. Green horizontal line denotes sellar pressure changes following Surgicel ${ }^{\mathrm{TM}}$ repair, blue is following dural substitute (Durepair ${ }^{\mathrm{TM}}$ ) repair and red is following NSF repair. The values in the yellow boxes denote the sellar pressure readings at which 'breach' occurred in the repaired defect for that particular repair technique. Absence of yellow boxes for a particular horizontal line denotes absence of breach in any of the CPAP settings tested for that particular repair technique (For instance in specimen 3, no breach was noted after Durepair ${ }^{\mathrm{TM}}$ and NSF repair). 
Fig 3 depicts the proportion of sellar pressure recorded following three repair techniques.

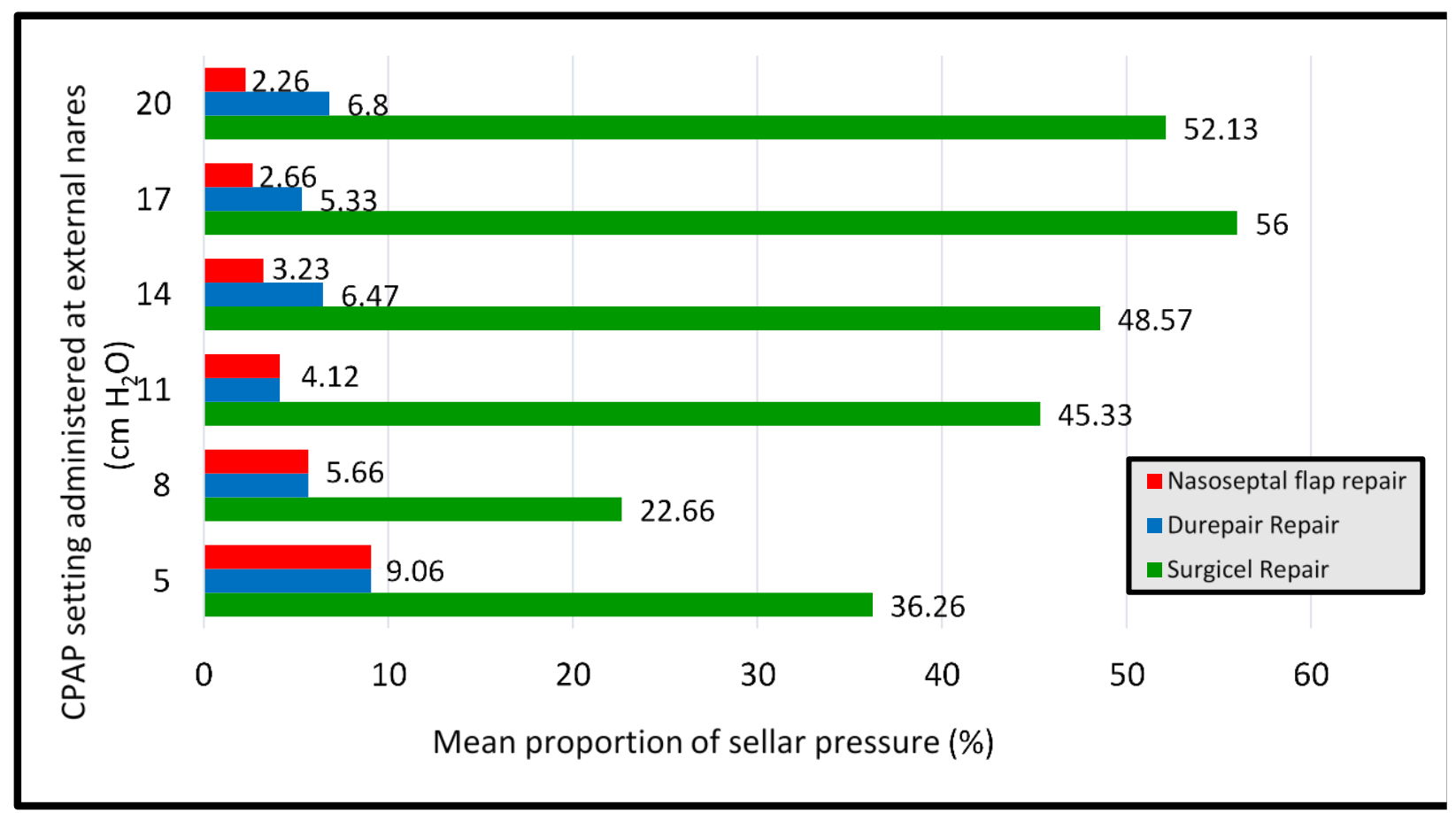

$\mathrm{X}$-axis determines the mean proportion of sellar pressure (in percentage) at each level of CPAP setting (considering respective CPAP setting delivered at the external nares as 100\%). Y-axis denotes different CPAP settings tested $(5-20 \mathrm{~cm} \mathrm{H} 2 \mathrm{O})$. The green bar represents mean proportion of sella pressure following Surgice $\left.\right|^{T M}$, the blue bar is following dural substitute (Durepair ${ }^{\mathrm{TM}}$ ) and red bar, following NSF repair techniques. 